\title{
The chemotaxonomic classification of Rhodiola plants and its correlation with morphological characteristics and genetic taxonomy
}

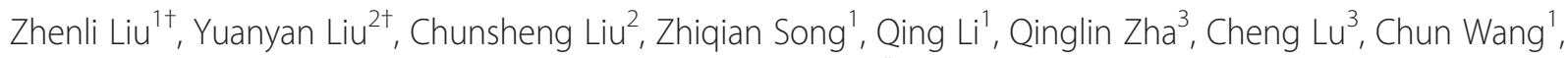
Zhangchi Ning ${ }^{2}$, Yuxin Zhang ${ }^{2}$ Cheng Tian ${ }^{2}$ and Aiping $\mathrm{Lu}^{3,4^{*}}$

\begin{abstract}
Background: Rhodiola plants are used as a natural remedy in the western world and as a traditional herbal medicine in China, and are valued for their ability to enhance human resistance to stress or fatigue and to promote longevity. Due to the morphological similarities among different species, the identification of the genus remains somewhat controversial, which may affect their safety and effectiveness in clinical use.

Results: In this paper, 47 Rhodiola samples of seven species were collected from thirteen local provinces of China. They were identified by their morphological characteristics and genetic and phytochemical taxonomies. Eight bioactive chemotaxonomic markers from four chemical classes (phenylpropanoids, phenylethanol derivatives, flavonoids and phenolic acids) were determined to evaluate and distinguish the chemotaxonomy of Rhodiola samples using an HPLC-DAD/UV method. Hierarchical cluster analysis (HCA) and principal component analysis (PCA) were applied to compare the two classification methods between genetic and phytochemical taxonomy.
\end{abstract}

Conclusions: The established chemotaxonomic classification could be effectively used for Rhodiola species identification.

Keywords: Rhodiola plants, Morphological characteristic, Genetic taxonomy, Phytochemical taxonomy

\section{Background}

The genus Rhodiola L. (Crassulaceae) comprises approximately 96 species found in the alpine regions of Asia and Europe. A total of 73 species, 2 subspecies and 7 varieties are found in China [1,2]. Rhodiola species, historically used as adaptogens in Russia and northern Europe and as a traditional herbal medicine in China, are valued for their ability to enhance human resistance to stress or fatigue and to promote longevity [3-5]. Rhodiola plants are mainly distributed in southwest and northwest of China, with most species located in Tibet and in Sichuan province. In China, the Rhodiola species called Hongjingtian have been used as an important adaptogen, hemostatic, and tonic in traditional Tibetan medicines for thousands of years [6]. The

\footnotetext{
* Correspondence: lap64067611@126.com

${ }^{\dagger}$ Equal contributors

${ }^{3}$ Institute of Basic Research in Clinical Medicine, China Academy of Chinese Medical Sciences, Beijing 100700, China

${ }^{4}$ School of Chinese Medicine, Hong Kong Baptist University, Kowloon Tong, Hong Kong SAR, China

Full list of author information is available at the end of the article
}

phytochemical extracts of Rhodiola plants are widely used throughout Europe, Asia and the United States, with biological activities including anti-allergenic and antiinflammatory effects and enhanced mental alertness, as well as a variety of other therapeutic applications [5]. Because of their commercial utility, Rhodiola plants are now cultivated in many locations in Europe and Asia. Most notably, the roots and rhizomes of $R$. crenulata $(R C)$ have high activities and have been accepted by the Pharmacopoeia of China [7]. In addition, many Rhodiola plants, such as $R$. sachalinensis $(R S), R$. himalensis (D. Dons) S. H. Fu $(R H)$, $R$. serrata $\mathrm{H}$. Ohba (RSE), R. rosea $L$. $(R R), R$. kirilowii (Regel) Maxim (RK) and R. fastigiata (HK. F. et Thoma) S. $\mathrm{H}$. Fu $(R F)$, etc., are also used as Hongjingtian in China. However, the identification of the closely related species of Rhodiola plants is often difficult due to their generally similar morphology.

Phytochemical investigations show that there are six important classes of constituents in Rhodiola rhizomes, including phenylpropanoids, phenylethanol derivatives,
() Chemistry Central

(c) 2013 Liu et al.; licensee Chemistry Central Ltd. This is an Open Access article distributed under the terms of the Creative Commons Attribution License (http://creativecommons.org/licenses/by/2.0), which permits unrestricted use, distribution, and reproduction in any medium, provided the original work is properly cited. 
flavonoids, monoterpernes, triterpenes and phenolic acids [8-10]. Using animal models, bioassay-guided fractionation of various extracts of plant adaptogens have shown that the active components are mainly phenylpropanoids and phenylethanol derivatives, including salidroside, rosavin and tyrosol [4,11-15]. In the Chinese Pharmacopoeia, salidroside is chosen as a marker compound for quality control [7]. Phenylpropanoids, such as rosarin, rosavin and rosin, are not only typical for Rhodiola rhizomes but are also pharmacologically active as antioxidants and neuro-stimulants [16-18]. Compounds such as tyrosol and gallic acid had been proven to be good radical scavengers $[19,20]$. A recent study revealed that rhodionin and salidroside might have anti-tumor effects $[21,22]$. Additionally, rhodionin is recognized to be involved in learning and memory [23,24]. The above 8 compounds from four chemical classes were selected as chemotaxonomic markers in the present paper. Because they exhibit variety bioactivities, meanwhile, phenylpropanoids and phenylethanol derivatives are characteristic in Rhodiola plants. Therefore, it is worthwhile to study the variety of co-existing phytochemical constituents in the plant, which may be responsible for its unique pharmacological activity.

The current taxonomical status of the genus Rhodiola has become quite complex. The rationale and defining criteria for the boundaries of the genus remain somewhat controversial [25]. The morphologies of different species of commercial Rhodiola plants are too similar to distinguish visually. With the development of DNA sequencing methods and the discovery of the polymerase chain reaction (PCR) for DNA amplification, biological systematic analysis has increasingly been based on DNA sequence analysis. The genotypes identified by PCR amplification suffice to predict the species of plants. In addition to genetic taxonomy and other classical morphological and non-morphological methods, phytochemical taxonomy can also provide supplementary information in species identification [26]. The chemotype of a plant species has traditionally been defined as by profile of natural products, and the genotype has been defined as its genetic constitution or DNA sequence.

Here, we collected 47 Rhodiola samples used clinically in different provinces of China. They were identified through morphological characterization and genotyping. In addition, eight bioactive compounds from four chemical classes (phenylpropanoids, phenylethanol derivatives, flavonoids and phenolic acids) were used as chemotaxonomic markers to elucidate the phytochemical taxonomy by an HPLC-DAD/UV method.

\section{Experimental}

\section{Chemicals and materials}

Methanol and acetonitrile were purchased from Fisher Scientific (Pittsburgh, PA, USA). The other reagents were from Beijing Chemical Inc. (Beijing, China). Gallic acid, salidroside and $(+)$ catechin standards were purchased from the National Institutes for Food and Drug Control (Beijing, China). Tyrosol, rhodionin, rosavin, rosarin and rosin standards were obtained from Chromadex (Irvine, CA, USA). The chemical structures of the eight reference marker compounds are listed in Figure 1.

The 47 authentic Rhodiola samples were collected from thirteen local provinces of China: Gansu, Jiangxi, Qinghai, Zhejiang, Heilongjiang, Henan, Hebei, Sichuan, Beijing, Neimeng, Liaoning, Xizang and Xinjiang. They were identified as genuine samples of $R$. crenulata $(R C)$, $R$. sachalinensis $(R S), R$. himalensis (D. Dons) S. H. Fu $(R H)$, R. serrata H. Ohba (RSE), R. rosea L. $(R R)$, R. kirilowii (Regel) Maxim (RK) and R. fastigiata (HK. F. et Thoma) S. H. Fu $(R F)$ by Professor Chunsheng Liu. Dried voucher specimens (marked as $R C-1 \sim R C-19, R S-20 \sim R S-21$, $R H-22, R S E-23 \sim R S E-37, R F-38, R R-39$ and $R K-40 \sim R K-47)$ were deposited at the Institute of Basic Theory, China Academy of Chinese Medical Sciences, Beijing, P. R. China, as shown in Table 1.

\section{HPLC-DAD/UV analysis}

The data were obtained using an Agilent 1100 Series HPLC with DAD. The analytical conditions for recording chromatograms of the marker compounds in Rhodiola samples were as follows. A Zobax SB-C 18 column (4.6 mm× $150 \mathrm{~mm}, 5 \mu \mathrm{m}$; Agilent Technologies) was used. The mobile phase consisted of $\mathrm{MeCN}$ (A) and $0.2 \%$ HAc $(B)$ with a linear gradient elution at a flow rate of $1.0 \mathrm{~mL} / \mathrm{min}$. The gradient program $(\mathrm{A} / \mathrm{B}, \mathrm{v} / \mathrm{v})$ was as follows: 5:95 $(\mathrm{t}=0 \mathrm{~min}), 8: 92(\mathrm{t}=10 \mathrm{~min}), 18: 82(\mathrm{t}=43 \mathrm{~min})$ and $37: 63(t=60 \mathrm{~min})$. The detection wavelength program was $275 \mathrm{~nm}(\mathrm{t}=0 \mathrm{~min}), 250 \mathrm{~nm}(\mathrm{t}=30 \mathrm{~min})$ and $332 \mathrm{~nm}(\mathrm{t}=45 \mathrm{~min})$. The column temperature was set to

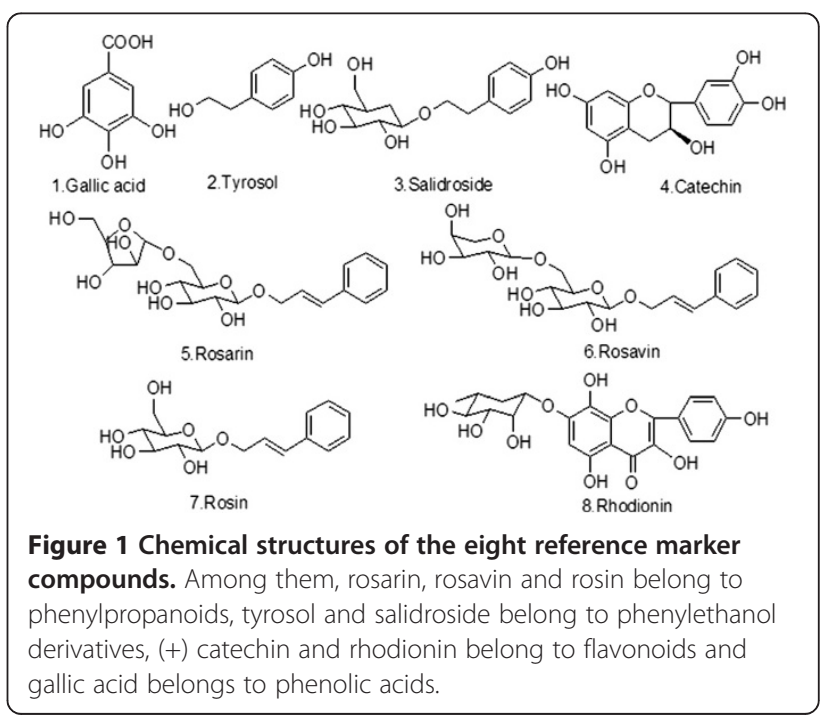


Table 1 The origins of the 47 Rhodiola samples

\begin{tabular}{lllllllll}
\hline Name & NO. & Location & Name & NO. & Location & Name & NO. & Location \\
\hline RC 1 & H7 & Gansu & RC 17 & H24 & Beijing & RSE 33 & H17 & Xizang \\
RC 2 & H2 & Jiangxi & RC 18 & H23 & Beijing & RSE 34 & H25 & Xizang \\
RC 3 & H5 & Jiangxi & RC 19 & H32 & Beijing & RSE 35 & H31 & Hebei \\
RC 4 & H9 & Gansu & RS20 & - & Liaoning & RSE 36 & Beijing \\
RC 5 & H4 & Jiangxi & RS21 & - & Heilongjiang & RSE 37 & H27 & Beijing \\
RC 6 & H6 & Gansu & RH22 & H13 & Henan & RF38 & Beijing \\
RC 7 & H3 & Jiangxi & RSE23 & H10 & Neimeng & RR39 & - & Xinjiang \\
RC 8 & H1 & Jiangxi & RSE 24 & H11 & Neimen & RK 40 & - & Qinghai \\
RC 9 & - & Qinghai & RSE 25 & H14 & Liaoning & RK 41 & - & Qinghai \\
RC 10 & - & Zhejiang & RSE 26 & H8 & Gansu & RK 42 & - & Qinghai \\
RC 11 & - & Heilongjiang & RSE 27 & H18 & Xizang & RK 43 & H34 & Qinghai \\
RC 12 & - & Henan & RSE 28 & H19 & Xizang & RK 44 & H33 & Qinghai \\
RC 13 & - & Gansu & RSE 29 & H20 & Xizang & RK 45 & H35 & Qinghai \\
RC 14 & - & Hebei & RSE 30 & H21 & Xizang & RK 46 & - & Qinghai \\
RC 15 & H29 & Sichuan & RSE 31 & H22 & Xizang & RK 47 & - & Qinghai \\
RC 16 & H30 & Hebei & RSE 32 & H16 & Xizang & &
\end{tabular}

$40^{\circ} \mathrm{C}$. The detection wavelength was selected by DAD according to max UV absorption of each reference.

\section{Sample preparation}

The samples were pulverized, and the powder (1.0 g) was accurately weighed and extracted with $25 \mathrm{~mL}$ of methanol by ultrasonication for $30 \mathrm{~min}$. After cooling, the solution was filtered through a $0.2 \mu \mathrm{m}$ membrane filter and stored at $4^{\circ} \mathrm{C}$ until analysis. A $5-\mu \mathrm{L}$ aliquot solution was injected for HPLC analysis. Each sample was prepared in triplicate and relative standard deviation (RSD) was calculated for all the samples.

\section{Method validation}

The eight standards were prepared and serially diluted with methanol to obtain seven different concentrations used for plotting standard curves, respectively. Method precision was determined by injecting one Hongjingtian sample solution six times consecutively. Reproducibility was studied through six independently prepared samples from a single batch of Hongjingtian. The stability test was performed by successively injecting the same sample solution over 24 hours. The limit of detection (LOD) and limit of quantity (LOQ) were determined at a signal-to-noise ratio (S/N) of 3 and 10, respectively. Standard solutions were diluted to series of appropriate concentrations with methanol and $5 \mu \mathrm{L}$ aliquots of the diluted solutions were injected into the HPLC for analysis.

\section{DNA extraction}

Nucleic acids were extracted and purified from deepfrozen plant materials. Sample vouchers were deposited in the collection at Beijing University of Chinese Medicine. Genomic DNA was extracted from silica gel-dried root material using a plant DNA extraction kit (Tiangen, Beijing, China) according to the manufacturer's protocol with some modifications. The quality of the isolated DNA was verified from absorbance measurements at wavelengths 230, 260 and $280 \mathrm{~nm}$ and on a 1\% (w/v) ethidium bromide-stained agarose gel.

\section{PCR, cloning and sequencing}

The PCR procedure was designed according to the instruction manual of a GeneRacer Kit (Invitrogen, Carlsbad, CA, USA). To achieve the $5^{\prime}$-end cDNA sequence, two rounds of thermal asymmetric interlaced PCR were performed as described in Liu and Chen et al. [27]. All of the obtained fragments were sequenced in both directions by ligating into pGEM-T vector and using an ABI 3730XL Genetic Analyzer (Applied Biosystems). PCR was performed using a touchdown strategy: $94^{\circ} \mathrm{C}$ for $4 \mathrm{~min}$, followed by $10 \mathrm{cy}-$ cles of $94^{\circ} \mathrm{C}$ for $75 \mathrm{~s}, 53^{\circ} \mathrm{C}$ for $5 \mathrm{~min}, 0.2^{\circ} \mathrm{C} / \mathrm{s}$ to $41^{\circ} \mathrm{C}$, and $72^{\circ} \mathrm{C}$ for $5 \mathrm{~min}$, followed by 35 cycles of $94^{\circ} \mathrm{C}$ for $1 \mathrm{~min}$, $45^{\circ} \mathrm{C}$ for $2 \mathrm{~min}$, and $72^{\circ} \mathrm{C}$ for $5 \mathrm{~min}$. The PCR products were run on a $1 \%(\mathrm{w} / \mathrm{v})$ ethidium bromide-stained agarose gel with a $6 \times$ orange loading buffer (Fermentas, Vilnius, Lithuania). The expected size band (680 bp) was excised from the gel and eluted using a Qiaquick Gel Extraction kit from Qiagen. The eluted PCR product was cloned into pGEM-T Easy Vector (Promega Corporation, Madison, USA) and sequenced using the BigDye Terminator Cycle Sequencing Kit (PE Applied Biosystems, Warrington, UK). The full-length deduced amino acid sequence was aligned with the publicly available HQT groups using ClustalX and 
MEGA version 4.0 software. A neighbor-joining (NJ) tree was constructed based on standard parameters with bootstrap testing of 1000 replicates. All the DNA sequences obtained were submitted to GenBank-NCBI for comparison with the deposited sequences using the tool BLAST [28].

\section{Statistical and multivariate analysis}

The statistical analysis was performed using the SAS 9.1.3 statistical package (order no. 195557) for PCA and HCA. PCA and HCA were used to show the unsupervised clustering pattern of the Rhodiola species. PCA and HCA were used to observe the natural interrelationships among the chemical components for each of the Rhodiola samples. The critical $p$ value for all analyses in this study was set to 0.05 .

\section{Results and discussion}

\section{Morphological characteristic of the collected samples}

The 47 collected Rhodiola samples were identified according to their morphological characteristics, and their collection locations are listed in Table 1. According to Flora of China [2], the morphological characteristic of Rhodiola plants is as following: stems dimorphic with usually very stout caudex or rhizome, usually with brown or blackish, membranous, scalelike leaves, sharply differentiated from much more slender, erect or ascending, leafy flowering stems. The roots and rhizomes of Rhodiola are used as the medicinal parts of the plants. The supplementary characteristic information from the aerial parts of the collected samples could not be obtained in this experiment. The morphologies of some Rhodiola samples are too similar to distinguish visually. In addition, the current taxonomical status of the genus Rhodiola has become quite complex. Accordingly, the species of some samples were tentatively identified. Among the 47 Rhodiola samples, $R C$ $1 \sim R C$-19 were identified as $R$. crenulata; $R S-20 \sim R S-21$ were identified as $R$. sachalinensis; $R H-22$ was identified as $R$. himalensis (D. Dons) S. H. Fu; RSE-23 RSE-37 were identified as $R$. serrata $\mathrm{H}$. Ohba; $R F-38$ was identified as $R$. fastigiata (HK. F. et Thoma) S. H. Fu; $R R-39$ was identified as $R$. rosea L.; $R K-40 \sim R K-47$ were identified as $R$. kirilowii (Regel) Maxim, as shown in Table 1.

\section{Genetic taxonomic of the collected samples}

To verify the accuracy of the identity of the species of the 47 Rhodiola samples, a phylogenetic tree was constructed based on the DNA sequences obtained from GenBank. For some samples, it was difficult to extract the exact DNA when the roots had been stored too long and were very dry, even when the roots were contaminated by microbes or the DNA was degraded. Therefore, only 34 Rhodiola samples were identified according to their DNA sequences. Among them, 23 Rhodiola samples were unambiguously identified with the similarities higher than $98 \%$. The other 11 Rhodiola samples were tentatively identified. All sequences were submitted to GenBank (http://www.ncbi.nlm.nih.gov/genbank/) and their IDs were shown in the Additional file 1.

HCA was employed to analyze the genetic data to characterize the population genetics of the Rhodiola samples from different geographic regions and determine their genetic diversity and population differentiation (Figure 2a). Glycyrrhiza astragalina was selected as an out-group to obtain more precise branching of the phylogenetic tree. The Rhodiola populations sampled from China were grouped separately according to their genotype. To avoid subjective errors by operator, the samples were relabeled as $\mathrm{H} 1 \sim \mathrm{H} 35$. The NJ tree obtained from the 23 Rhodiola samples was divided into five main characteristic branches. The first branch consisted of $R C$. The second branch included $R S E$. The first branch $(R C)$ progressively merged with the second branch (RSE) to form a larger cluster that merged with the third branch $(R F)$. The fourth branch $(R H)$ and the fifth branch $(R K)$ together with the above three branches created the whole tree structure.

\section{Phytochemical taxonomy of the collected samples}

Based on the morphological characteristic and genetic taxonomy, both of them have some limitations in species identification. The classification of plants based on chemotypes can be used as a powerful chemotaxonomic tool that provides a detailed view of the differences and similarities between species. The 8 pure bioactive compounds classified into four types were used as chemotaxonomic markers to distinguish among the different Rhodiola samples.

\section{Method validation}

To gain high sensitivity and good peak capacity, the chromatographic conditions were optimized, as described in the HPLC-DAD/UV analysis section. MeCN and $0.2 \%$ HAc were used as the mobile phase to improve the retention behavior of the constituents on the HPLC column. The wavelength for the detection of compounds was selected by DAD. The chromatograms at $275 \mathrm{~nm}$ could provide maximum absorption of gallic acid, tyrosol, salidroside and (+) catechin. The wave length for the detection of rosarin, rosavin and rosin was at $250 \mathrm{~nm}$, and $332 \mathrm{~nm}$ was used for the detection of rhodionin.

The method was validated in terms of linearity, LOD and LOQ, precision, reproducibility, stability and recovery test, the results of which are shown in Table 2. Calibration curves were prepared by plotting the peak areas of the marker compounds versus the corresponding concentrations. Good linear relationships $\left(R^{2}=1\right.$ for rhodionin and 0.9999 for tyrosol, salidroside, rosarin, rosavin, rosin, (+) catechin and gallic acid) were demonstrated over a range of $0.071-737 \mu \mathrm{g} / \mathrm{mL}$. The accuracy of the analytical method 


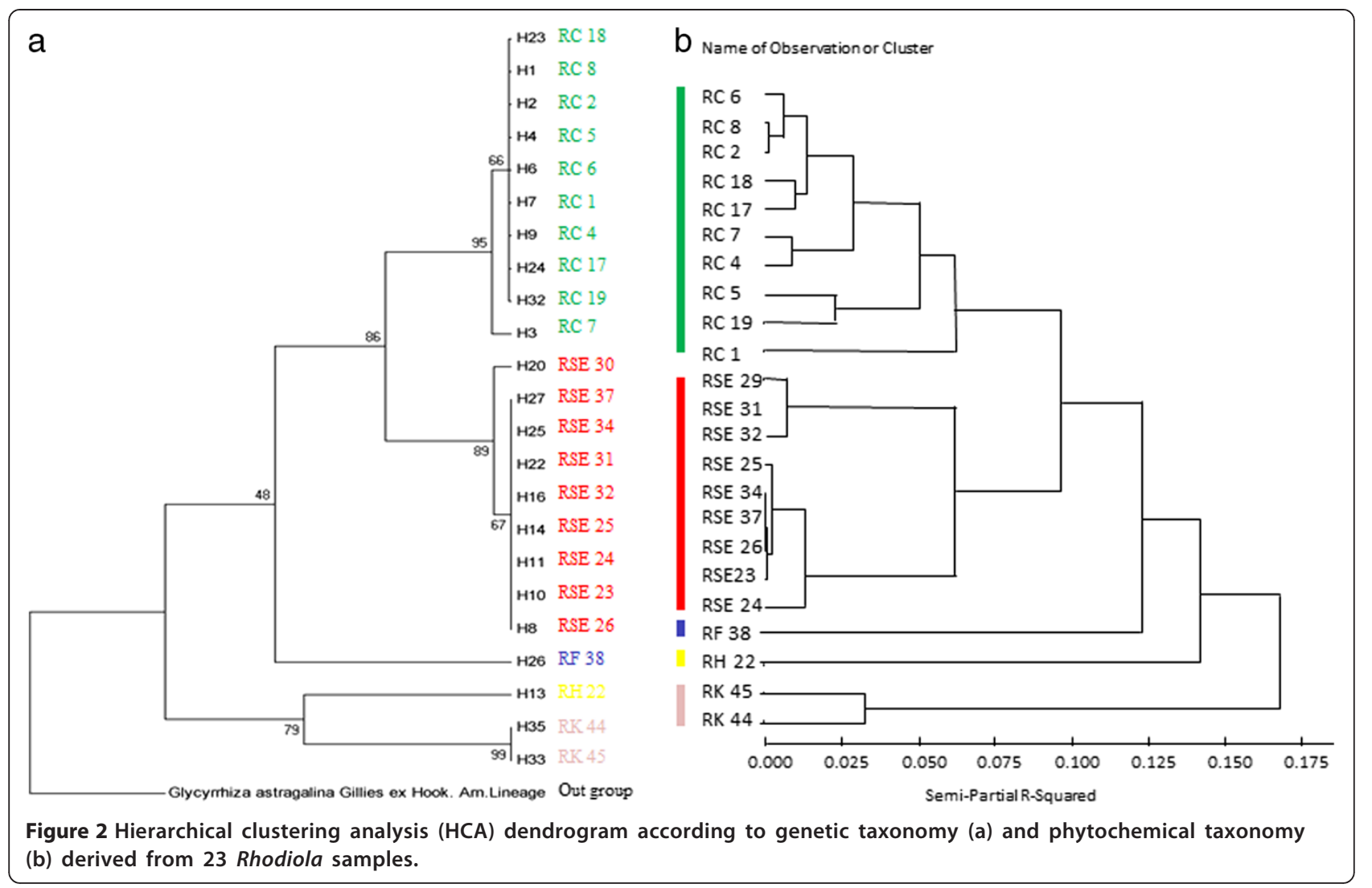

was evaluated using a recovery test. The mean recoveries were from 97.5 to $101.7 \%$ with RSD less than $3.0 \%$ for the eight reference compounds. The precision of the assay was determined by its reproducibility. The RSD of peak area ranged from 0.49 to $2.47 \%$.

According to the validated method described above, the 8 reference compounds could be visually distinguished from each other, and all the peaks were simultaneously eluted within 55 minutes. The representative chromatograms of each Rhodiola species and the eight standard mixtures were provided in Figure 3. It indicated that different kinds of reference markers occurred at various concentrations in the different Rhodiola species of Hongjingtian samples. For example, gallic acid and salidroside could be detected in all species. Rosarin and rosin was characteristic in $R S, R H$ and $R R$ species, which was consistent with the reported literature $[5,11]$. Rhodionin could barely be detected in $R H$ and $R K$ species. Rosavin was existed only in $R H, R S E$ and $R R$ species. The contents of reference compounds were determined for the 47 Rhodiola samples, as shown in Table S1 in Additional file 1.

Table 2 Linear regression, LODs and LOQs, precisions, reproducibility, stability and recovery for eight compounds

\begin{tabular}{|c|c|c|c|c|c|c|c|c|c|c|}
\hline \multirow{2}{*}{ Compound } & \multirow{2}{*}{$\begin{array}{c}\text { Regression } \\
\text { equation } \\
(n=2)\end{array}$} & \multirow[t]{2}{*}{$r$} & \multirow{2}{*}{$\begin{array}{l}\text { Linear range } \\
(\mu \mathrm{g} / \mathrm{mL})\end{array}$} & \multirow{2}{*}{$\begin{array}{c}\text { LOD } \\
(\mu \mathrm{g} / \mathrm{mL})\end{array}$} & \multirow{2}{*}{$\begin{array}{c}\text { LOQ } \\
(\mu \mathrm{g} / \mathrm{mL})\end{array}$} & \multirow{2}{*}{$\begin{array}{c}\text { Precision } \\
(n=6) \text { RSD (\%) }\end{array}$} & \multirow{2}{*}{$\begin{array}{l}\text { Reproducibility } \\
(n=6) \text { RSD (\%) }\end{array}$} & \multirow{2}{*}{$\begin{array}{l}\text { Stability } \\
\text { (RSD,\%) }\end{array}$} & \multicolumn{2}{|c|}{ Recover $(n=6)$} \\
\hline & & & & & & & & & Recovery (\%) & RSD (\%) \\
\hline Salidroside & $Y=218.51 X-0.4$ & 0.9999 & $3.50-700$ & 0.089 & 0.27 & 0.95 & 1.46 & 0.53 & 99.22 & 2.45 \\
\hline Tyrosol & $Y=384.13 X-3.9$ & 0.9999 & $0.74-737$ & 0.014 & 0.043 & 2.67 & 2.42 & 2.40 & 101.7 & 1.68 \\
\hline Gallic acid & $Y=2923.6 X-13.8$ & 1 & $2.52-504$ & 0.042 & 0.13 & 0.49 & 1.80 & 1.88 & 98.44 & 2.85 \\
\hline$(+)$ Catechin & $Y=339.77 X+3.7$ & 0.9999 & $3.25-325$ & 0.031 & 0.15 & 1.87 & 2.35 & 2.11 & 99.69 & 2.25 \\
\hline Rosarin & $Y=1954.5 X-2.7$ & 0.9999 & $3.21-321$ & 0.094 & 0.36 & 1.90 & 2.05 & 1.67 & 99.77 & 2.80 \\
\hline Rosavin & $Y=2140.4 X+8.4$ & 0.9999 & $0.071-284$ & 0.0078 & 0.027 & 1.97 & 1.66 & 1.96 & 97.51 & 2.25 \\
\hline Rosin & $Y=3076.8 X-4.2$ & 0.9999 & $1.25-375$ & 0.037 & 0.18 & 1.45 & 1.19 & 1.10 & 100.5 & 2.77 \\
\hline Rhodionin & $Y=166.15 X+1.3$ & 0.9999 & $0.18-183$ & 0.0094 & 0.032 & 2.32 & 2.12 & 2.61 & 99.4 & 1.72 \\
\hline
\end{tabular}




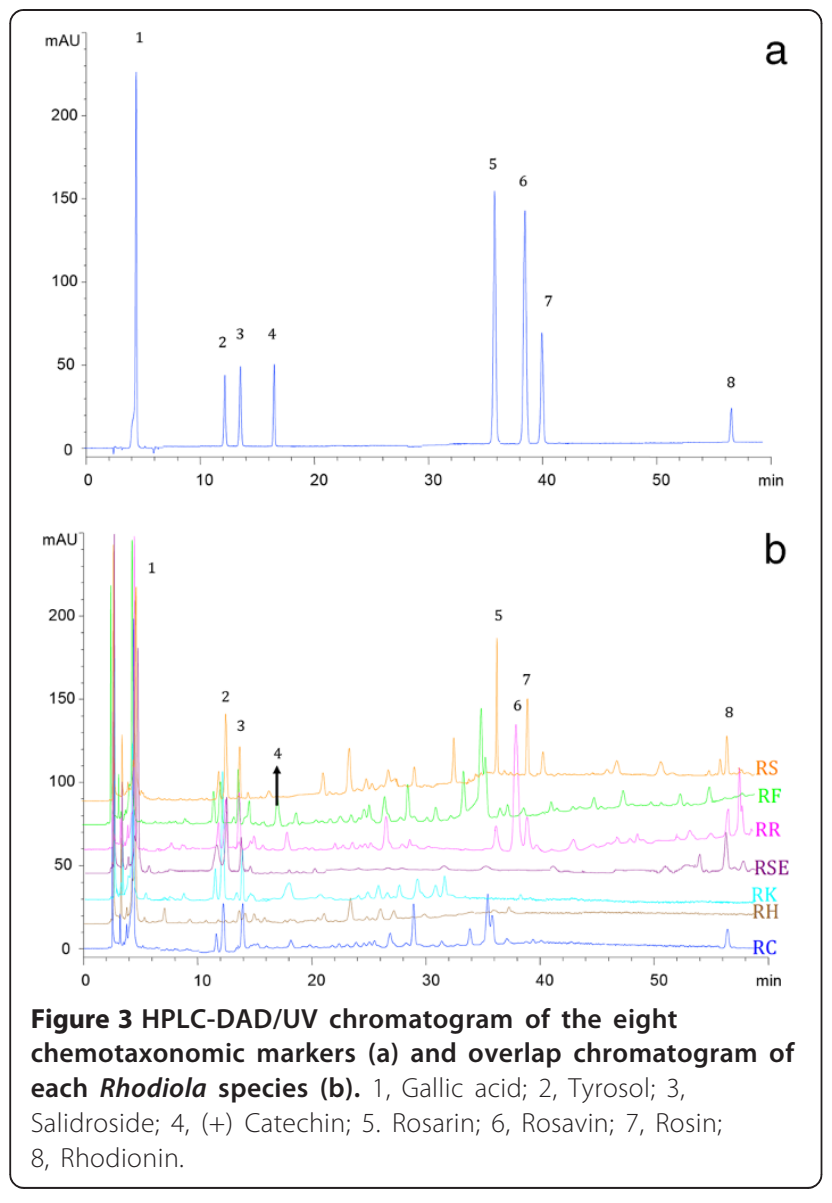

Accordingly, the 23 Rhodiola samples classified by genetic taxonomy were analyzed by chemotaxonomic classification using the four types of bioactive compound as reference markers. HCA showed that Rhodiola samples were divided into five branches according to their chemotaxonomy (Figure 2b). Of the two classification methods for classifying the genus Rhodiola samples, HCA results showed considerably comparable results for both the genotype- and chemotype-based classification methods. This observation may be because different genotypes caused different chemotypes due to the genotype-dependent production of metabolites.

Furthermore, to differentiate all the Rhodiola samples by chemotaxonomic classification, an unsupervised pattern recognition method (PCA) was performed. A twocomponent PCA score plot of HPLC-DAD/UV data was utilized to depict the general variation of the marker references among the 47 Rhodiola samples. As shown in Figure $4 \mathrm{a}$, the samples were primarily divided into four clusters according to their species. The clustering pattern observed from the HCA tree (Figure $4 \mathrm{~b}$ ) is consistent with the PCA. According to the PCA and HCA tree, samples $R K 43$ and $R C 11$ and 12 were distinctively excluded from their original branches, which were merged in the $R C$ and $R S E$ species, respectively. It indicated that samples of $R K 43$ and $R C 11$ and 12 identified by morphological characteristics are not correct. The present chemotaxonomic classification could be effectively used for Rhodiola species identification.
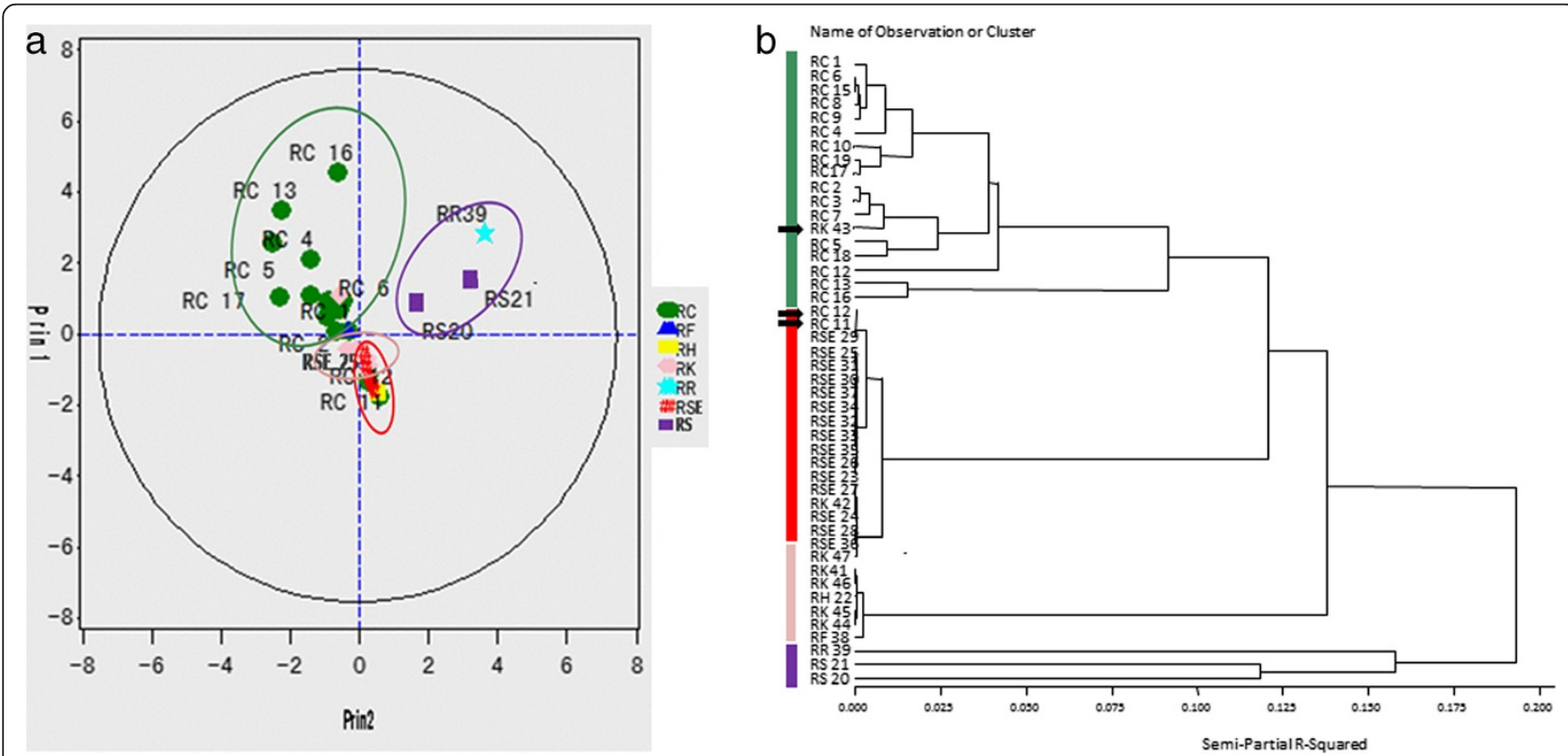

Figure 4 Principal component analysis (PCA) score plot (a) and hierarchical clustering analysis (HCA) dendrogram (b) derived from the HPLC-DAD/UV data set of the Rhodiola samples. 


\section{Conclusions}

In this study, the 47 Rhodiola samples used commercially in China were identified by their morphological characteristics and genetic and phytochemical taxonomies. In the morphological characteristics, there exists variation between populations of the same species at different life stages and from different environments [29]. If the samples collected are not intact, the accuracy of the identification will be affected. The morphologies of some Rhodiola samples are too similar to distinguish visually. Genetic taxonomy can provide exact classification of species submitted to GenBank. However, there may be uncertainty in the extraction of pure DNA from every sample, and the procedure of analyses is timeconsuming. Here, eight bioactive compounds from four chemical classes (phenylpropanoids, phenylethanol derivatives, flavonoids and phenolic acids) were used as chemotaxonomic markers to evaluate and distinguish the chemotypes of 47 Rhodiola samples by an HPLCDAD/UV method. First, 23 Rhodiola samples classified by genetic taxonomy and morphological characteristics were analyzed by chemotaxonomic classification, which showed considerably comparable results. This analysis indicated that different genotypes caused different chemotypes due to the genotype-dependent production of metabolites. Next, all the 47 Rhodiola samples were analyzed by PCA and HCA based on the content of the eight bioactive references. All the samples were divided into four clusters according to the established phytochemical taxonomic method. Consequently, chemotyping became useful for distinguishing morphologically similar species, by identifying variants of the chemotaxonomic markers. However, certain limitations exist in the present studies. The number of collected samples from $R S, R H, R F$ and $R R$ species is low, which affected the accuracy of this phytochemical taxonomic classification. Moreover, the clusters of genes for biosynthesis regulating the metabolite profiling need to be investigated in future.

\section{Additional file}

Additional file 1: The DNA sequences and their IDs of the detected samples were provided in the Supplementary Materials. The contents of reference compounds from 47 Rhodiola samples were listed in Table 1S.

\section{Competing interests}

The authors declare that they have no competing interests.

\section{Authors' contributions}

LA provided the concept and designed the study. LZ and LY conducted the analyses and wrote the manuscript. LC, SZ, LQ, ZQ, LC, WC, NZ, ZY and TC participated in the research. All authors have read and approved the final manuscript.

\section{Acknowledgements}

This study was financially supported by the National Science Foundation of China (Project No. 30772726, No. 30825047, No. 30902000 and No. 81001623).

\section{Author details}

'Institution of Basic Theory, China Academy of Chinese Medical Sciences, Beijing, China. ${ }^{2}$ School of Chinese Materia Medica, Beijing Municipal Key Laboratory for Basic Research of Chinese Medicine, Beijing University of Chinese Medicine, Beijing, China. ${ }^{3}$ Institute of Basic Research in Clinical Medicine, China Academy of Chinese Medical Sciences, Beijing 100700, China. ${ }^{4}$ School of Chinese Medicine, Hong Kong Baptist University, Kowloon Tong, Hong Kong SAR, China.

Received: 18 March 2013 Accepted: 10 July 2013

Published: 12 July 2013

\section{References}

1. Li T, Zhang $\mathrm{H}$ : Identification and comparative determination of rhodionin in traditional tibetan medicinal plants of fourteen Rhodiola species by high-performance liquid chromatography-photodiode array detection and electrospray ionization-mass spectrometry. Chem Pharm Bull(Tokyo) 2008, 56:807-814.

2. Fu SX: Flora of China. Beijing: Science Press; 1984.

3. Mattioli L, Titomanlio F, Perfumi M: Effects of a Rhodiola rosea L. extract on the acquisition, expression, extinction, and reinstatement of morphineinduced conditioned place preference in mice. Psychopharmacology 2012, 221:183-193.

4. Mattioli L, Funari C, Perfumi M: Effects of Rhodiola rosea L. extract on behavioural and physiological alterations induced by chronic mild stress in female rats. J Psychopharmacol 2009, 23:130-142.

5. Tolonen A, Hohtola A, Jalonen J: Comparison of electrospray ionization and atmospheric pressure chemical ionization techniques in the analysis of the main constituents from Rhodiola rosea extracts by liquid chromatography/mass spectrometry. J Mass Spectrom 2003, 38:845-853.

6. Yang YCHTN, Lu SL, Hung RF, Wang ZX: Zang Yao Zhi. Xining: Qinghai People's Publishing House; 1991.

7. Pharmacopoeia: Pharmacopoeia of the People's Republic of China. 144th edition. Beijing: Chemical Industry Press; 2010.

8. Ali Z, Fronczek FR, Khan IA: Phenylalkanoids and monoterpene analogues from the roots of Rhodiola rosea. Planta Med 2008, 74:178-181.

9. Panossian AWG, Sarris J: Rosenroot (Rhodiola rosea): traditional use, chemical composition, pharmacology and clinical efficacy. Phytomedicine 2010, 7:481-493.

10. Hohtola A: Bioactive compounds from northern plants. Adv Exp Med Biol 2010, 698:99-109.

11. Kurkin VA, Zapesochnaya GG: Chemical composition and pharmacological properties of Rhodiola rosea L. Chemical and Pharmaceutical Journal 1986, 20:1231-1244

12. Zapesochnaya GG, Kurkin VA, Boyko VP, Kolkhir VK: Phenylpropanoids promising biologically active compounds of medicinal plants. Khim Farm Zh 1995, 29:47-50

13. Peschel W, Prieto JM, Karkour C, Williamson EM: Effect of provenance, plant part and processing on extract profiles from cultivated European Rhodiola rosea L. for medicinal use. Phytochemistry 2012, 86:92-102.

14. Cifani C, Micioni Di BM, Vitale G, Ruggieri V, Ciccocioppo R, Massi M: Effect of salidroside, active principle of Rhodiola rosea extract, on binge eating. Physiol Behav 2010, 101:555-562.

15. Wiedenfeld $H$, Dumaa M, Malinowski M, Furmanowa M, Narantuya S: Phytochemical and analytical studies of extracts from Rhodiola rosea and Rhodiola quadrifida. Pharmazie 2007, 62:308-311.

16. Huang SC, Lee FT, Kuo TY, Yang JH, Chien CT: Attenuation of long-term Rhodiola rosea supplementation on exhaustive swimming-evoked oxidative stress in the rat. Chin J Physiol 2009, 52:316-324.

17. Schriner SE, Abrahamyan A, Avanessian A, Bussel I, Maler S, Gazarian M, Holmbeck MA, Jafari M: Decreased mitochondrial superoxide levels and enhanced protection against paraquat in Drosophila melanogaster supplemented with Rhodiola rosea. Free Radic Res 2009, 43:836-843

18. Kucinskaite A, Briedis V, Savickas A: Experimental analysis of therapeutic properties of Rhodiola rosea L. and its possible application in medicine. Medicina (Kaunas) 2004, 40:614-619.

19. Sun L, Isaak CK, Zhou Y, Petkau JC OK, Liu Y, Siow YL: Salidroside and tyrosol from Rhodiola protect $\mathrm{H} 9 \mathrm{c} 2$ cells from ischemia/reperfusioninduced apoptosis. Life Sci 2012, 91:151-158. 
20. Lee MW, Lee YA, Park HM, Toh SH, Lee EJ, Jang HD, Kim YH: Antioxidative phenolic compounds from the roots of Rhodiola sachalinensis A. Bor. Arch Pharm Res 2000, 23:455-458.

21. Sun C, Wang Z, Zheng Q, Zhang H: Salidroside inhibits migration and invasion of human fibrosarcoma HT1080 cells. Phytomedicine 2012, 19:355-363.

22. Hu X, Lin S, Yu D, Qiu S, Zhang X, Mei R: A preliminary study: the antiproliferation effect of salidroside on different human cancer cell lines. Cell Biol Toxicol 2010, 26:499-507.

23. Choe Kl, Kwon JH, Park KH, Oh MH, Kim MH, Kim HH, Cho SH, Chung EK, Ha SY, Lee MW: The antioxidant and anti-inflammatory effects of phenolic compounds isolated from the root of rhodiola sachalinensis a. BOR. Molecules 2012, 17:11484-11494.

24. Kobayashi K, Yamada K, Murata T, Hasegawa T, Takano F, Koga K, Fushiya S, Batkhuu J, Yoshizaki F: Constituents of Rhodiola rosea showing inhibitory effect on lipase activity in mouse plasma and alimentary canal. Planta Med 2008, 74:1716-1719.

25. de Kuppler AL, Steiner U, Sulyok M, Krska R, Oerke EC: Genotyping and phenotyping of Fusarium graminearum isolates from Germany related to their mycotoxin biosynthesis. Int J Food Microbiol 2011, 151:78-86.

26. Desjardins AE: Natural product chemistry meets genetics: when is a genotype a chemotype. J Agric Food Chem 2008, 56:7587-7592.

27. Liu YG, Chen Y: High-efficiency thermal asymmetric interlaced PCR for amplification of unknown flanking sequences. Biotechniques 2007 43:649-650. 652, 654 passim.

28. Altschul SF, Madden TL, Schaffer AA, Zhang J, Zhang Z, Miller W, Lipman DJ: Gapped BLAST and PSI-BLAST: a new generation of protein database search programs. Nucleic Acids Res 1997, 25:3389-3402.

29. Sandasi M, Kamatou GP, Viljoen AM: An untargeted metabolomic approach in the chemotaxonomic assessment of two Salvia species as a potential source of alpha-bisabolol. Phytochemistry 2012, 84:94-101.

doi:10.1186/1752-153X-7-118

Cite this article as: Liu et al:: The chemotaxonomic classification of Rhodiola plants and its correlation with morphological characteristics and genetic taxonomy. Chemistry Central Journal 2013 7:118.

\section{Publish with ChemistryCentral and every scientist can read your work free of charge \\ "Open access provides opportunities to our colleagues in other parts of the globe, by allowing anyone to view the content free of charge." \\ W. Jeffery Hurst, The Hershey Company. \\ - available free of charge to the entire scientific community \\ - peer reviewed and published immediately upon acceptance \\ - cited in PubMed and archived on PubMed Central \\ - yours - you keep the copyright \\ Submit your manuscript here: \\ http://www.chemistrycentral.com/manuscript/<smiles>c1ccccc1</smiles> \\ Chemistry Central}

